\title{
Comunicación y educación: una alianza necesaria en el contexto de la sociedad del siglo XXI
}

\author{
Verónica Verdugo Bonvallet*
}

\section{RESUMEN}

En este trabajo se presentan los principales resultados de una investigación desarrollada con jóvenes universitarios de la Universidad Católica Silva Henríquez durante el año 2004. Dado que se ubica en la perspectiva de conocer las subjetividades puestas en juego en el proceso de situarse en el rol de estudiante universitario, los objetivos de esta investigación se orientaron a conocer los significados que los estudiantes atribuyen al proceso de enseñanza aprendizaje', los espacios y prácticas comunicativas formales y no formales que valoran en tanto instancias de aprendizaje y su inserción en los medios de comunicación masiva y las nuevas tecnologías de la comunicación y la información como espacios de significación.

Palabras clave: Proceso enseñanza aprendizaje / Significados / Prácticas comunicativas

\section{ABSTRACT}

This article illustrates the main results of a research study carried out with young people from the Catholic University Silva Henríquez during the year 2004. Since it adopts the perspective of trying to find out the subjectivities at hand in the process of positioning itself in the role of the university student, the goals of this research were oriented to the meaning that the students gave to the teaching - learning process, the spaces and formal as well as non-formal communicative practices, the instances of learning and the insertion in mass media along with the new technologies of communication and information as places of significance.

Key words: Teaching - learning process / meanings / communicative practices

Chilena. Trabajadora Social. Académica del Departamento de Trabajo Social. Universidad Católica Silva Henríquez. E-mail: vverdugo@ucsh.cl

En el contexto de este trabajo entendemos que la aproximación a la forma en que los estudiantes significan su proceso de enseñanza aprendizaje, supone la comprensión de los significados que atribuyen a la institución universitaria, a la noción de aprendizaje, a la figura del docente, a sí mismos, a sus pares y al aula. 


\section{Antecedentes}

E n la actualidad, existe consenso en la centralidad de la educación para el desarrollo continuo de las personas y de la humanidad. En el contexto de las crecientes transformaciones del siglo XXI, la política educativa adquiere un rol protagónico no sólo en la generación de conocimientos o de la capacidad técnica de los países, sino también en el desarrollo de relaciones armoniosas entre individuos y naciones. A partir de la necesidad de encontrar respuestas en este campo problemático, en las últimas décadas se han venido desarrollando estudios desde distintas disciplinas y aproximaciones teórico metodológicas para su comprensión e interpretación.

Por otra parte, pese a la existencia de una vasta literatura e investigaciones empíricas que dan cuenta de los desafíos que la sociedad moderna plantea a la educación, en general el modelo clásico de educación continúa siendo hegemónico². La superación de esta aparente contradicción, a saber, la organización y funcionamiento de las instituciones educativas desde una lógica situada preferentemente en un modelo más tradicional que coexiste con un sinnúmero de señales que apuntan a una perspectiva crítica de la educación, pasa necesariamente por modificar una visión restrictiva de la educación en tanto "transmisora de conocimientos" y reconocer la manera en que los actores del proceso educativo se sitúan frente al mismo y el proceso comunicativo que allí tiene lugar. Ello supone la comprensión del sentido y alcance de la dimensión comunicativa presente en todo acto educativo, asumiendo la educación como un proceso continuo de negociación y construcción activa de significados histórica y socialmente situados.

Concebida en estos términos, la educación es esencialmente un proceso de comunicación, donde estudiantes y docentes entran en contacto y "participan en común" a través de múltiples formas

\footnotetext{
Al respecto, véase Martín-Barbero, Jesús "Oficio de Cartógrafo Travesías Latinoamericanas de la Comunicación en la Cultura”, capítulo "Desafíos Culturales de la Comunicación a la Educación"; Quiroz, María Teresa, "Aprendizaje y Comunicación en el siglo XXI", capítulo 3 "Educación, Cultura y Medios de comunicación", Brunner, José Joaquín: "Educación: Escenarios de Futuro. Nuevas Tecnologías y Sociedad de la Información" y Monereo Carles y Pozo Ignacio "La Universidad Ante la Nueva Cultura Educativa: Enseñar y Aprender para la Autonomía", capítulol La Cultura Educativa en la Universidad: Nuevos Retos para Profesores y Alumnos.
} 
de expresión que, de manera simultanea, transitan desde el "cara a cara" propio de la comunicación interpersonal, hasta intercambios comunicativos tecnológicamente mediados.

En dicho proceso, continuo e integral, se ponen en práctica las distintas dimensiones presentes en la comunicación humana, que hacen posible que, mediante el lenguaje verbal y no verbal, docentes y estudiantes construyan cotidianamente códigos comunicativos y estructuras de significación.

La consideración de estos aspectos, así como de la incidencia de los cambios sociales en el campo de la educación es fundamental, toda vez que éstos modifican la cultura de los actores y su relación con los sistemas en que éstos participan, en este caso, el educacional. En palabras de Barbero, resulta estratégico pensar la inserción de la educación en los complejos procesos de comunicación de la sociedad actual, escenario difuso de informaciones, lenguajes y saberes y descentrado por relación a los dos centros -la escuela ${ }^{3}$ y el libro- que organizan aún el sistema educativo vigente (Martín-Barbero, Jesús, 2002: 332)

El desconocimiento de la manera en que los jóvenes, en el marco de los cambios que ha experimentado la sociedad, negocian y reorganizan los significados propuestos por la cultura escolar y la institución en que reciben su formación, generaría una disonancia comunicativa entre los significados que la institución universitaria y sus estudiantes atribuyen al proceso de enseñanza aprendizaje, aspecto que incide directamente en los resultados de este último.

Respecto de este punto, desde la perspectiva de Quiroz, a lo largo del proceso de modernización en América Latina se produce una fractura que afecta en su esencia la relación educativa. Dicha fractura se generaría a partir de la escisión de la realidad, del discurso del maestro, generando dos culturas. La primera, puntualiza la autora, representa a la de la institución encarnada en los adultos, que se mueve con lentitud, propone un conocimiento arcaico y un modelo de vida en el que el tiempo se adensa, se solidifica y se apoltrona

Para Martín-Barbero la noción de escuela no remite a la educación primaria, sino a todo el sistema educativo que abarca desde la educación básica hasta la universidad. 
en el pasado. La segunda, la de los jóvenes, seguiría el ritmo de las zonas más modernizadas de la sociedad influidas por la ciencia y la tecnología, los medios electrónicos de comunicación, la computación y una vertiginosa movilidad espacial y de las relaciones sociales. Se trata de una cultura que valora el mundo real y concreto que habitan los jóvenes y en la que los sentimientos ocupan un lugar muy importante. (Quiroz, Teresa, 2003: 58)

Por su parte, Reguillo, siguiendo a Mead, señala que la sociedad está experimentando un nuevo momento cultural, caracterizado porque tanto el pasado como el presente se reconfiguran a partir de un futuro incierto. Desde la perspectiva de la autora, la consolidación y/o aceleración de una serie de tendencias -tales como, la mundialización de la cultura por la vía de las industrias culturales, los medios de comunicación y las super tecnologías de la información, el triunfo del discurso neoliberal montado sobre el adelgazamiento del Estado y sobre la exaltación del individualismo, el empobrecimiento estructural y creciente de grandes sectores de la población; el descrédito y deslegitimación de las instancias y dispositivos tradicionales de representación y participación- han afectado a los jóvenes en su percepción de la política, del espacio y del futuro. En este contexto, los jóvenes, pese a diferencias de clase, género y emblemas aglutinadores, compartirían las siguientes características de fin de siglo: Una conciencia planetaria, globalizada, la priorización de los pequeños espacios de la vida cotidiana como trincheras para impulsar la transformación global, un respeto por el individuo, que se convierte en el centro de sus prácticas; la selección cuidadosa de las causas en las que se involucran y la deslegitimación del barrio como centro del mundo y de sus prácticas (Reguillo, Roxana, 2000: 142 - 143).

Cabe destacar algunos antecedentes arrojados por la Tercera Encuesta Nacional de Juventud ${ }^{4}$ realizada en Chile, donde a modo general se establece que los jóvenes se atribuyen una distinción generacional al sentirse notoriamente diferentes a las generaciones juveniles pasadas y a los adultos. Respecto de éstos últimos, el estudio revela una visión crítica que los califica como incoherentes o mentirosos. Asimismo, reconocen una diversidad generacional in-

$4 \quad$ Instituto Nacional de la Juventud, Tercera Encuesta Nacional de Juventud, Capítulo 4 Orientaciones Culturales e Identidad Juvenil. 
terna en aspectos tales como el género, la posición social y la localización. Asignan importancia a los medios de comunicación en el uso de su tiempo libre, confían en la ecuación educación - empleo como fórmula para tener éxito en la vida y alcanzar la felicidad, se sitúan en una posición de apertura y respeto frente a las diferencias y opciones personales y dan cuenta de una capacidad mayor de reunificar en su visión de mundo y prácticas de vida, lo que habitualmente parece incompatible. Finalmente, es importante destacar que diversos antecedentes obtenidos a partir de este estudio revelan la predominancia de una orientación pragmática y moderna en las definiciones estratégicas que los jóvenes hacen.

La falta de conciencia en el sistema educativo de la escisión del discurso del maestro de la realidad a la que hacíamos referencia, se traduce en la atribución de la responsabilidad a los estudiantes de las dificultades que hoy enfrenta la educación. Por su parte, los estudiantes, herederos de una cultura audiovisual y para quienes el texto escrito y la universidad forman parte de un escenario diverso y complementario donde es posible adquirir aprendizajes, no siempre encuentran sentido a la propuesta educativa del maestro. En su experiencia, la figura del docente y la actividad pedagógica que se desarrolla en el aula no constituirían los únicos referentes, obteniendo aprendizajes diversos relacionados con experiencias comunicativas extra aula, en las que desarrollan habilidades y destrezas e incorporan conocimientos que integran en su formación. En este escenario, los medios de comunicación masiva y las nuevas tecnologías de la comunicación se han abierto espacio entre los jóvenes y formarían parte de sus prácticas comunicativas cotidianas.

Con relación a estos aspectos, Huergo señala que los medios y las nuevas tecnologías han operado cruciales transformaciones en nuestras percepciones y acciones, imaginario, modos de relacionarnos (la socialidad y la cotidianidad) y expectativas colectivas (Huergo, Jorge, 2000:9). Ello implica que el cambio tecnológico no puede ser concebido superficialmente como un mero acceso a aparatos modernos, el acento no está puesto en que los jóvenes disponen de computadores, Internet u otros dispositivos de información y comunicación, sino en los nuevos modos de ver, leer, pensar y aprender a que ello da origen. 
La consideración de estos antecedentes nos plantea la necesidad de vincular, en las coordenadas histórico sociales de la sociedad actual, la educación con la comunicación en la perspectiva de comprender el proceso de comunicación/educación implicado en la práctica pedagógica. Lo anterior, en tanto los significados que los jóvenes otorgan a la experiencia educativa, se relacionan íntimamente con la manera en que se relacionan y sitúan frente a la universidad, al docente, su grupo de pares y en definitiva, al proceso de enseñanza - aprendizaje de nivel universitario que los convoca.

\section{Enfoque metodológico}

Dado que el propósito de la investigación fue comprender los significados atribuidos por los jóvenes universitarios a su proceso de enseñanza aprendizaje, esta se enmarcó en el paradigma interpretativo y desarrolló una metodología cualitativa. Ello nos permitió investigar la realidad de los jóvenes en su contexto natural a partir de su propio discurso. La unidad de estudio estuvo conformada por estudiantes de carreras de pre grado de la Universidad Católica Cardenal Raúl Silva Henríquez, con una muestra de tipo no probabilística o dirigida. Las técnicas de recolección de información que se que utilizaron son: Focus group y Cuestionario electrónico estructurado.

\section{Supuestos, preguntas y objetivos de investigación}

El estudio se planteó como supuestos que los jóvenes poseen valoraciones y significaciones del espacio educativo y los actores allí presentes distintas a las que propone el sistema de educación superior y que dichas diferencias no han sido incorporadas por el sistema educativo. En cuanto a los objetivos de investigación, éstos se orientaron a caracterizar, desde una perspectiva educativo-comunicacional, la manera en que los jóvenes estudiantes de la UCSH significan su proceso de enseñanza aprendizaje. 


\section{Resultados de la investigación ${ }^{5}$}

\section{La Universidad}

Con respecto a los atributos de la institución universitaria se puede señalar que los estudiantes poseen una pluralidad de significaciones. Una tendencia del discurso la ve como fuente de obtención de beneficios o adquisiciones personales. La experiencia en la universidad proporciona a los jóvenes herramientas para ampliar su visión de mundo y desarrollarse como personas. Ello implica la incorporación de una amplia gama de aprendizajes y conocimientos que favorecen su relación con el medio social, con otros y consigo mismos. Así, en la experiencia de los jóvenes, la adquisición de conocimientos no tiene prioridad en detrimento de otras fuentes de aprendizaje y, por lo tanto, la experiencia educativa es concebida como un todo. Esta visión es coherente con la propuesta de Delors (1996), con relación a la demanda que las sociedades modernas plantean a la educación en términos de resignificarla, en una concepción más amplia orientada a promover en las personas el descubrimiento e incremento de sus posibilidades creativas. Otra mirada, presente en los jóvenes, destaca la dimensión colectiva de la universidad. Desde esta óptica, esta institución educativa se constituye en un espacio propicio para realizar proyectos orientados a favorecer el bien común. Las afirmaciones de los jóvenes dan cuenta de una postura crítica según la cual la universidad carece de sentido colectivo y, por lo tanto, no estaría respondiendo a las expectativas que ellos tenían a su ingreso, ni desarrollando una función que consideran le es propia. Se trata de una crítica dirigida fundamentalmente a los propios estudiantes, quienes se conducirían de acuerdo a una orientación más individualista que no recoge los intereses y nece-

Es importante señalar que en los focus group se conformó de un discurso homogéneo entre géneros. En la dinámica conversacional que asumió cada grupo fue posible distinguir temáticas que, más que responder a una diferenciación de género, eran representativas de la forma en que el grupo se posicionó frente al tema, distinguiéndose, en ocasiones, más de una postura y opiniones encontradas frente a un mismo tópico. Por otra parte, la convergencia del discurso de los jóvenes hizo posible la realización de un análisis simultáneo de los cinco grupos focales, estableciéndose, cuando corresponde, las distinciones que dan cuenta de la especificidad del discurso representativo de un área o nivel específico. Por otra parte, el análisis de la información obtenida a partir del cuestionario electrónico estructurado, se realiza en forma conjunta con aquella emanada de los grupos focales, en función de los ejes temáticos propuestos en un inicio. 
sidades presentes en la comunidad universitaria. Por otra parte, los "proyectos colectivos" a los que se alude, tal como plantea la Tercera Encuesta Nacional de Juventud, están referidos a instancias concretas y propias de su entorno inmediato. Es importante destacar que los principios que están a la base de la misión de la UCSH, no se verían reflejados en la experiencia que estos jóvenes han tenido respecto de la participación y promoción de iniciativas colectivas en la universidad. Finalmente, una tercera aproximación rescata el sentido instrumental de la universidad. En el contexto social actual, la obtención de un título profesional parece no solo necesaria sino también indispensable, en tanto permitiría acceder a nuevas posibilidades de inserción social y laboral. Con relación a este aspecto, es importante considerar los antecedentes relativos al perfil de los estudiantes de la UCSH, quienes en un gran porcentaje constituyen la primera generación que accede a la universidad en sus familias. No obstante lo anterior, los jóvenes tienen claro que poseer un título profesional constituye solo una "carta de presentación" que por sí sola no les asegura el éxito futuro.

Con relación a los elementos de identidad específica de la UCSH, es posible señalar que los jóvenes reconocen y valoran la vocación social que está a la base del proyecto institucional de la UCSH. Uno de los aspectos con los cuales se identifican es la orientación humana y cristiana que subyace a la formación que pretende impartir. Desde su óptica ello se expresa fundamentalmente en una perspectiva valórica que sitúa a la persona en el centro de atención y que se distingue claramente del proselitismo religioso. Este significado recoge no sólo los planteamientos de la misión institucional, sino también la significación que docentes, empleadores y egresados otorgan a esta casa de estudios. En el mismo sentido, otro elemento distintivo de la UCSH refiere al tipo de relación que se establece entre estudiantes y académicos, donde se reconoce a la persona como el eje fundamental de dicha relación. Se trata de relaciones humanas caracterizadas por la cercanía y el compromiso recíproco con el otro, desarrollándose un tipo de relación interpersonal que al legitimar la importancia del componente afectivo en la práctica pedagógica, rompe con la fragmentación clásica razón - emoción que sitúa a unos y a otros en posiciones perfectamente diferenciadas y en espacios excluyentes. Respecto de esta forma de situarse o, al decir de Reguillo (2000), de la actualización sujetiva que realizan los jóvenes a partir de la interiorización diferenciada de los esque- 
mas de la cultura vigente, es importante retomar el planteamiento de Quiróz (2003) con relación al lugar que ocupan los sentimientos en la cultura de los jóvenes. Particularmente, en el contexto de la $\mathrm{UCSH}$, la importancia y protagonismo que adquiere la persona del estudiante en esta relación es significado como una expresión del sello distintivo de esta casa de estudios, aspecto que representa una fractura con experiencias educativas anteriores.

\section{Concepción de aprendizaje}

La concepción de aprendizaje que representa la tendencia más generalizada en los jóvenes, se asocia con "aplicación". Para éstos la apropiación de conocimientos pasa necesariamente por la utilización de lo aprendido en nuevas situaciones, donde, a partir del saber hacer, evalúan los conocimientos adquiridos. Desde esta óptica el aprendizaje excede los límites del espacio académico ya que se proyecta y pone en práctica en contextos diversos, permitiéndoles elaborar nuevas ideas y conductas. Esta definición recoge los tres rasgos del "buen aprender" que propone Pozo en su análisis sobre el concepto de aprendizaje, quien señala que este debiera producir cambios, posibilitar la utilización de lo aprendido en nuevas situaciones y generarse siempre producto de la práctica, aspectos que constituirían la base de la apropiación en los educandos (Pozo, Ignacio, 1996: 75 - 82 )

Por otra parte, para los estudiantes la fuente de legitimación de dicha adquisición no depende de una figura externa, ni de un mecanismo formal, es el propio joven el encargado de discernir. La fijación de la evaluación en el propio estudiante, da cuenta del reconocimiento implícito de lo que Fontcuberta, siguiendo a Linard denomina "autonomía del aprendizaje" cuando destaca la capacidad del estudiante de definir, tanto sus objetivos de aprendizaje, como los caminos para alcanzarlos (Fontcuberta, Mar, 2003).

Otra tendencia del discurso, estrechamente ligada con la anterior, está representada por una concepción de aprendizaje como desarrollo personal. Desde esta mirada, la adquisición de aprendizajes por parte del educando fija en él un cambio personal que le permite alcanzar nuevos niveles de desarrollo. Así, la evaluación de aprendizajes esta mediada por una expresión concreta del crecimiento que se genera en la persona que lo incorpora. 


\section{El docente en el proceso de enseñanza aprendizaje}

La concepción de los roles y relaciones que docentes y estudiantes tienen o pueden tener en la práctica pedagógica que los convoca, es flexible. Para los jóvenes el proceso de enseñanza aprendizaje se configura a partir de relaciones e interacciones entre sujetos que poseen saberes y capacidad dialógica en una práctica comunicativa caracterizada por la horizontalidad. Se trata de una concepción de educación como proceso comunicacional que trasciende la concepción tradicional que atribuye el patrimonio del conocimiento al docente y que abre nuevas posibilidades a formas diversas de interacción y comunicación. Al dar cuenta de la centralidad del tipo de comunicación presente en el proceso educativo, esta perspectiva, releva la noción de comunicación educativa propuesta por Kaplún, para quien la comunicación se constituye en un componente pedagógico (Kaplún, Mario, 1998).

La existencia de cercanía en la relación que se establece entre docentes y estudiantes representa una tendencia predominante en el discurso de los jóvenes. Este aspecto es altamente valorado y reconocido como propio de la UCSH. Dicha cercanía no sólo se traduce en el establecimiento de relaciones pedagógicas más personalizadas, sino también en la ausencia de una división marcada en los estudiantes entre el ámbito personal y académico. Los jóvenes no se relacionan de manera disociada con la universidad. En su experiencia, emoción y razón, aparecen integrados como parte de una misma realidad, lo que permite a estos últimos recibir apoyo frente a problemáticas que emergen de otros contextos e inciden directamente en su rendimiento académico. Desde esta perspectiva, se aprecia una simetría "implícita" en las expectativas que cada uno de estos actores tienen respecto del tipo de relación que esperan entablar. Por una parte, los estudiantes reivindican el componente afectivo en las relaciones académicas y, por otra, los académicos acogen activamente dicha demanda. Dichas expectativas son consonantes con los principios valorados en el contexto institucional donde se desarrolla la relación.

Por otra parte, el dominio de los contenidos específicos de la cátedra es considerado fundamental, tanto en la valoración que se hace del docente, como en la adquisición de aprendizajes. Se trata de una concepción dinámica según la cual el conocimiento ha de 
ser contextualizado y renovado en función de los nuevos aprendizajes adquiridos en el proceso de enseñanza aprendizaje, así como de la consideración del escenario societal en que dicho proceso se genera. Esta perspectiva deja atrás la concepción escolarizante de educación y sitúa al "maestro" en un modelo de enseñanza y en un rol que se aproxima a lo que Pozo reconoce en la sociedad actual como "cultura del aprendizaje", entendiendo por tal a un proceso permanente y dinámico que, entre otras cosas, se renueva e integra distintos escenarios, contextos y necesidades de aprendizaje.

Para los estudiantes que finalizan su formación en carreras del área educación la labor del docente no se limita a la transmisión de contenidos. Cumple la función de educar y lo hace a partir de la forma en que asume el proceso de enseñanza aprendizaje del estudiante. El docente se constituye en un modelo que educa con su actitud ante la cátedra que imparte, el estudiante y el proceso que quiere intencionar. Ello supone, por un lado, la atribución de potencial educativo a la amplia gama de comportamientos verbales y no verbales presentes en la práctica pedagógica (los cuales incluso pueden llegar a ser más significativos que los saberes específicos de la cátedra que se imparte) y, por otro, da cuenta de un proceso de comunicación y educación permanente que opera en un contexto relacional específico, en distintos niveles, donde los actores construyen activamente y en conjunto, significados en torno a la experiencia educativa.

\section{El Estudiante en el proceso de aprendizaje}

Para los jóvenes la universidad representa un quiebre con su experiencia escolar formal, donde desaparece la figura de un agente externo que los impulse y controle. En este contexto, resignifican el rol que como estudiantes han venido desarrollando e inician un proceso orientado a la definición y cumplimiento de metas personales. Lo anterior supone la adquisición de un sentido de responsabilidad en un proceso de formación que integra el mundo académico con su participación en otros escenarios de aprendizaje, como el familiar o el de su grupo de pares. No obstante lo anterior, existe una visión que se contrapone a la resignificación del rol del estudiante que los jóvenes declaran, relacionada con el predominio de una cultura escolar en la universidad. A partir de ésta los fines de la educación aparecen más ligados a un modelo que releva el cumplimiento y certificación de etapas sucesivas, que a la generación de un proceso 
autonomía y desarrollo de capacidades en los estudiantes. En este modelo, la adquisición de aprendizajes y la fijación de metas personales por parte del estudiante son sustituidas por la importancia atribuida a la calificación y a la figura del docente como agente externo de evaluación. Esta visión es coherente con la apreciación de los docentes respecto de la dimensión escolarizante que es posible advertir en el estilo relacional docente - estudiante que la UCSH genera.

\section{Disonancia de significados atribuidos al proceso de aprendizaje}

A la base del proceso de comunicación presente en la práctica pedagógica está la coexistencia de dos códigos diferenciados que generan disonancias en la comunicación entre docentes y estudiantes. El lenguaje académico o, al decir de Bernstein, código "elaborado" que utilizarían los docentes sería aquel legitimado en el proceso de enseñanza aprendizaje de los estudiantes, mientras que el lenguaje cotidiano o código "restringido" al que se inclinarían de manera natural los jóvenes sería desestimado por situarse fuera de los cánones que valida el sistema de educación superior como adecuado. Dado que el código elaborado supone un mayor grado de complejidad para los estudiantes, éstos utilizan el restringido para comunicarse de manera cotidiana en los distintos espacios en que se mueven, hecho que les genera dificultades a la hora de enfrentar la relación pedagógica. Esta situación se hace particularmente compleja en las evaluaciones, donde sienten que, muchas veces, el docente fija su atención más en la forma (cómo se expresa) que en el fondo (los contenidos que comunica). Siguiendo a Bernstein ello da cuenta de la jerarquía de formas de comunicación esperables en este terreno, así como de los criterios por los que se rigen. A este respecto, los jóvenes demandan a los docentes la capacidad de distinguir entre apropiación de aprendizajes y código empleado para darlos a conocer. (Bernstein, Basil, 2001)

Con relación a este tema, el discurso de los jóvenes revela un doble perfil que tiende a ser contradictorio. Por una parte, significan el lenguaje académico como "el apropiado al contexto universitario" y consideran que los estudiantes tendrían que manejar este código ya que es coherente con la forma en que "debiera" darse la comunicación en este espacio, es decir, ellos incorporan en su discurso los 
significados que propone, reconoce y valida el sistema de educación superior. Por otra, en la práctica, emplean el lenguaje cotidiano en actividades académicas, demandando a los docentes la capacidad de entender y aceptar la forma de comunicación significada como propia de los jóvenes.

Es importante señalar, que los jóvenes desarrollan un proceso de auto reflexión que les permite evaluar por sí mismos la adquisición de aprendizajes. En este proceso la nota se torna secundaria y, muchas veces adquiere un sentido netamente instrumental. De igual manera, como ya se indicó, debido a que uno de los factores que media en la obtención de un alto o bajo rendimiento es la capacidad de dar cuenta de los aprendizajes al docente, los jóvenes sienten que el fracaso en la evaluación se relaciona directamente con la dificultad para adquirir una lógica formal abstractiva propia de la cultura del docente, que les permita organizar coherentemente, por escrito, los conceptos y aprendizajes obtenidos. En este proceso, la diferencia de códigos lingüísticos a las que alude Bernstein se torna central en la valoración que otorga el docente al contenido expresado por el estudiante, asumiendo centralidad la "forma" en que es expresado más que el contenido mismo. Así, los jóvenes organizan su formación estableciendo prioridades y mecanismos de adaptación para convivir con una propuesta educativa que no siempre les resulta fácil incorporar.

Es así como los jóvenes advierten la existencia de un modelo de comunicación pedagógica, mecánico y unidireccional al momento de la evaluación que los sitúa en lo que Kaplún (1998a) denomina un rol pasivo en su proceso de formación. En dicho modelo son los docentes quienes detentan un saber que admite una lectura unívoca, según la cual el estudiante ha de reproducir los contenidos que le fueron transmitidos. Los jóvenes se relacionan crítica y analíticamente con esta realidad resignificándola en términos pragmáticos de la siguiente forma. Por una parte, aprenden a reconocer las tendencias en el pensamiento de sus docentes, así como las expectativas que éstos tienen frente a los estudiantes y, por otra, responden los instrumentos de evaluación de acuerdo a las claves que aseguren su éxito académico aunque no legitimen al docente y su propuesta programática. De esta forma, se gesta una disonancia comunicativa en el sistema evaluativo que atenta contra los objetivos de aprendizaje y somete a "engaño" a sus protagonistas. 
En el discurso de los estudiantes una de las razones que explican la asimetría comunicativa generada a partir del uso de códigos distintos entre docentes y estudiantes se relaciona directamente con su experiencia escolar formal. Desde esta óptica, el problema de fondo se sitúa en el estudiante, en el pasado y la escuela. La dificultad para incorporar el lenguaje propio del mundo académico se originaría en la familiarización con un modelo de educación y comunicación lineal, que organizaría la actividad escolar fundamentalmente desde la transmisión y memorización de contenidos. En este contexto, la lectura y el libro propiamente tal, aparecen asociados a la educación formal, como un mecanismo de reproducción de contenidos, más que como un medio para la reflexión y la expresión de la creatividad que exceda los límites de la institución educativa. Finalmente, pese a que en el discurso de los jóvenes aparece como "deseable" la incorporación de un código que los sitúe en mejores condiciones para el logro del éxito académico, éstos no dan cuenta en su discurso de la incorporación de prácticas orientadas a la consecución de dicha aspiración.

\section{Relación docente - estudiante: un marco para la interlocución}

Los estudiantes que cursan carreras del área educación visualizan una práctica educativa que no siempre genera vías horizontales de interlocución. Tal como ocurre con la evaluación, se desarrolla así una relación docente - estudiante donde este último se reconoce como perteneciente a un espacio social relacional que hace impracticable un intercambio comunicativo orientado a negociar los sentidos, saberes y discursos de cada uno de los actores implicados. En este contexto, el maestro no llega a conocer el pensamiento del estudiante y, por lo tanto, prevalece una práctica comunicativa coherente con el modelo de pedagogía clásica según el cual existe una sola fuente legitimada del saber. Desde esta perspectiva, la libertad y autonomía que el estudiante requiere para relacionarse proactivamente con su maestro supone una transformación en las relaciones sociales presentes entre unos y otros.

\section{Esfuerzo V/S rendimiento académico}

Por otra parte, los estudiantes que finalizan su formación en el área de ciencias sociales enfatizan la rentabilidad del tiempo a la hora 
de organizar sus actividades académicas. Insertos en un contexto histórico social marcado por la rapidez, la eficiencia y la celeridad de los cambios, evalúan la "inversión" de este recurso en función de los resultados que esperan obtener. Esta perspectiva valida la obtención de metas a un mínimo costo, así como la utilización de estrategias orientadas a la aprobación de asignaturas con independencia del dominio de contenidos. Ello adquiere particular relevancia si se considera que la evaluación de la adquisición de aprendizajes no descansa en factores externos al estudiante y que la calificación, como se indicó, puede tornarse instrumental. Esta perspectiva es coherente con las orientaciones pragmáticas y modernas que, de acuerdo a la Tercera Encuesta Nacional de Juventud, se observaría en las definiciones estratégicas que los jóvenes hacen.

\section{Motivación del estudiante}

Otra tendencia del discurso, releva la motivación del estudiante como factor trascendental a la hora de decidir la "forma" en que se cursará una asignatura. Los jóvenes no significan de manera homogénea su carga académica, sino que a partir de una serie de factores -de índole personal y social- establecen prioridades y estrategias a seguir que permiten situar las distintas asignaturas en categorías diferenciadas -obtención de créditos, adquisición de aprendizajes, avanzar en el tiempo predeterminado en el programa curricular de la carrera, entre otros. Por otra parte, aún cuando el docente y su propuesta programática logren captar el interés del estudiante, dicha motivación no siempre sobrepasaría los límites de la asignatura.

\section{Presión por el éxito en la sociedad}

Finalmente, a la base de las decisiones que toman los estudiantes para cumplir metas académicas estaría el peso de la sociedad moderna que impone el éxito como mecanismo de integración social. Los jóvenes se sienten impelidos a obtener buenos resultados en las actividades que emprenden, objetivo que ya no es significado solo como producto del esfuerzo y del respeto por las reglas del juego. A este respecto, la obtención de logros con mínimo esfuerzo aparece en el discurso de los jóvenes como una práctica extendida en los estudiantes de la universidad que, al situar la certificación en un lugar trascendental, no es cuestionada. Asumido en estos términos, el tránsito de los estudiantes por la universidad, se torna 
complejo toda vez que se trata de un proceso en el que concurren factores de orden social e individual, que en combinaciones diversas y en distintos momentos del proceso, estarían dando cuenta de las distintas estrategias empleadas por los jóvenes en el camino hacia su titulación.

\section{Espacios y prácticas comunicativas formales que tienen valor de aprendizaje}

El aula aparece en el discurso de los jóvenes como el espacio social formal por excelencia para la comunicación y el aprendizaje. En ella se desarrollan prácticas comunicativas diversas entre estudiantes y entre éstos y sus maestros que "pueden" potenciar procesos reflexivos en los jóvenes y ser significativos en el proceso de enseñanza aprendizaje. Ello implica que no tiene valor en sí misma, sino que "depende" de una multiplicidad de factores que la legitiman ante el estudiante. Desde esta perspectiva, aspectos como la valoración del aporte del grupo de pares, la formación profesional de los docentes y su forma de impartir docencia, los contenidos temáticos, la motivación del estudiante y, en general, la dinámica que se da entre los distintos actores, se tornan significativos a la hora de atribuirle valor como lugar de aprendizaje. En este sentido, el aula como espacio de aprendizaje lejos de estar condenada a la "desaparición" por la emergencia de nuevos escenarios educativos, continúa siendo legitimada por los jóvenes, quienes demandan una postura creativa y renovada del docente frente a la práctica educativa.

En el discurso de los jóvenes el aula se presenta como un espacio privilegiado para la adquisición de aprendizajes cuyo eje principal se articula en torno al diálogo. En esta puesta en común los jóvenes activan procesos reflexivos que se extienden más allá de los límites físico-temporales de la sala de clases, permitiéndoles profundizar sus conocimientos. En dicho proceso, las palabras, las claves no verbales presentes en la comunicación humana, así como la ubicación espacial del estudiante en el aula, son concebidos como un todo integrado que da cuenta del complejo proceso de comunicación que allí se genera. Desde esta mirada, existe una crítica a la concepción clásica de educación que se inscribe en un modelo informacional de la comunicación. 


\section{Espacios y prácticas no formales que tienen valor de aprendizaje}

La práctica comunicativa no formal que predomina en el discurso de los jóvenes como fuente principal de adquisición de aprendizajes esta dada por el grupo de pares. Los jóvenes desarrollan una multiplicidad de prácticas en diferentes espacios dentro y fuera de la universidad, donde la comunicación de los estudiantes entre sí constituye un componente central de su proceso de enseñanza aprendizaje. Dichas prácticas comunicativas están al servicio de objetivos académicos y personales. Por otra parte, existe una diversidad de espacios significados por los jóvenes como instancias complementarias de aprendizaje, que podrían organizarse en dos categorías: Aquellos ubicados al interior del recinto educativo como el patio central, la plazoleta, el casino y, aquellos que habitan los jóvenes fuera de la universidad, donde desarrollan diversos tipos de relaciones con personas y grupos también diversos como la familia, el "carrete" o los distintos escenarios de participación en que se comprometen. Los jóvenes desarrollan un proceso de intercambio comunicativo en el que la puesta en común permite complementar y validar los aprendizajes adquiridos. En dicho proceso, la expresión y el diálogo se constituyen en un componente pedagógico central que posibilita la comprensión. Esto quiere decir que logran organizar y clarificar sus aprendizajes al convertirlos en un producto comunicable y hacer efectiva dicha comunicación. En esta dinámica, el grupo de pares es legitimado como un interlocutor válido para la adquisición e integración de aprendizajes provenientes de los distintos escenarios que los jóvenes habitan. El aprendizaje entonces es para los jóvenes un producto social. Asimismo, más que un hecho aislado, el trabajo colectivo constituye una práctica comunicativa informal permanente y generalizada que forma parte de su rutina académica. Con relación a este aspecto, cabe recordar el planteamiento de Kaplún (1998b) respecto de la relación existente entre el acceso de los sujetos que aprenden a amplias y variadas redes de interacciones comunicativas y los resultados de la educación. Por otra parte, el lenguaje cotidiano que los jóvenes utilizan en éstos intercambios favorece la comprensión y asimilación de contenidos.

Un segundo especio no formal que se presenta en su discurso como una práctica comunicativa que favorece la adquisición de aprendizajes es el "carrete". En este espacio, lo académico y lo lúdico se 
integran en un todo que hace posible la síntesis de lo aprendido. Se trata de un intercambio comunicativo de carácter simétrico donde el diálogo y la interacción posibilitan la construcción y apropiación de conocimientos. Por otra parte, no se atribuye al aula y la universidad el monopolio en la adquisición de aprendizajes, concibiéndolas como espacios que, junto a otros, hacen aportes distintos y complementarios.

\section{Proceso de aprendizaje $y$ TICs}

Una primera constatación que surge del análisis global de los tres ámbitos definidos inicialmente en la investigación -Internet, televisión y prensa escrita-, es que en el discurso de los jóvenes Internet aparece como la tecnología que representa el mayor aporte a su proceso de enseñanza aprendizaje y al que le dedican más tiempo.

No es posible establecer una categoría única respecto de los usos y significaciones de Internet por parte de los estudiantes. Aunque esta herramienta se ha abierto espacio entre los jóvenes y forma parte de sus prácticas comunicativas cotidianas, su empleo no es homogéneo y da cuenta de la integración de las dimensiones educativa, lúdica y comunicativa en una misma práctica. En el discurso predominante, Internet aparece como una herramienta altamente utilizada y valorada por los estudiantes. El acceso a la red les ofrece posibilidades de comunicarse, obtener, procesar y almacenar información que ellos aprovechan no sólo con fines académicos.

La comunicación e información de acceso fácil, actualizada e instantánea les permite rentabilizar sus tiempos, trabajar simultáneamente desde distintas ubicaciones espaciales y establecer vinculaciones con sus grupos de pares. Internet se constituye entonces en un medio para la comunicación, donde no solo se transmite información, sino que se gesta una conversación integrada en las prácticas comunicativas cotidianas que realizan los jóvenes particularmente con sus grupos de pares. La apropiación que los jóvenes tienen de Internet confirma los planteamientos de Hopenhayn (2003) con relación a la necesidad de pensar una educación para los cambios culturales y las nuevas formas que adquiere la información y la comunicación. En este caso, la comprensión de la forma en que los jóvenes aprenden y se comunican resulta vital para potenciar sus procesos de enseñanza aprendizaje. Uno de los aspectos importan- 
tes a considerar para el logro de este objetivo es la existencia de una concepción más amplia del aprendizaje, que integra lo lúdico y que no lo concibe únicamente como producto legítimo de la enseñanza formal universitaria. Se trata de un aprendizaje fundado en la propia exploración de los jóvenes, menos dependiente de figuras adultas como la del docente.

Finalmente, en el uso de la red digital coexisten lógicas distintas relacionadas no solo con los objetivos de aprendizaje que se plantean los estudiantes, sino también con fines más instrumentales como la obtención de calificaciones o aprobación de asignaturas. El uso instrumental de Internet constituye una alternativa válida que se pone en práctica frente a "determinadas circunstancias" que ponen en riesgo el éxito académico del estudiante y, por lo tanto, se legitima como una práctica que permite cumplir con el requerimiento de una determinada asignatura, más que como una alternativa real para el aprendizaje. Frente a este tema, como a otros, no hay una posición única y es posible advertir la diversidad interna presente en los jóvenes, a la que alude Reguillo (2000) y que queda de manifiesto en la Tercera Encuesta Nacional de Juventud realizada en nuestro país (1999). Entre estas posiciones es posible advertir un uso instrumental que incorpora los significados del sistema universitario, así como un uso instrumental significado como una alternativa más, cuyo ámbito de decisión compete única y exclusivamente al estudiante.

Para los estudiantes el rol del docente en el proceso de aprendizaje es insustituible, ello en tanto el acceso y uso de Internet por sí solo no garantiza la adquisición de conocimientos. Frente a la intensidad de la producción del saber y gran cantidad de información que circula por la red, la clase presencial tradicional se legitima como un espacio privilegiado para la interlocución. En esta óptica, el rol del docente se desplazaría desde la concepción tradicional de suministrar instrucción, a un rol de mediador de un proceso de enseñanza aprendizaje autónomo que emerge de las diferentes interacciones, fuentes, relaciones y referentes donde participan los jóvenes. A partir de dicho rol, el docente tendría que proporcionar las claves para seleccionar y comprender críticamente la información disponible.

Pese a que Internet es utilizado como apoyo a la docencia, los estudiantes no perciben en los docentes una incorporación generalizada 
e intencionada de esta tecnología, que evidencie el reconocimiento de su potencial educativo. Las prácticas docentes con relación a esta herramienta dan cuenta de un uso incipiente, de un desconocimiento de las amplias y variadas posibilidades que ella ofrece, así como de una desconfianza frente al uso que los estudiantes le puedan dar. Con relación a esto último, los alumnos declaran que la copia textual de la información que proporciona la red, aparecería como el principal temor por parte de los docentes.

La desconfianza que algunos docentes tienen con relación al potencial educativo de Internet, esta directamente vinculada con su uso instrumental. A la base de dicha apreciación estaría la idea de que Internet más que facilitar el proceso de enseñanza aprendizaje, permitiría a los estudiantes un acceso fácil y carente de contenido al logro de metas académicas. Esta visión crítica se vería reforzada por la existencia de prácticas concretas, que aunque no serían generalizadas en los estudiantes, pondrían de manifiesto la racionalidad instrumental con que los jóvenes se conducen frente a Internet.

\section{Proceso de aprendizaje y Medios de comunicación}

Con relación a la oferta programática de la televisión aparecen dos posiciones, una que sitúa la discusión en el receptor y otra que la ubica en el medio. Desde la primera, el discurso releva la necesidad de constituirse en receptores críticos de los medios de comunicación. Desde esta perspectiva el estudiante se concibe como un sujeto activo, crítico y reflexivo ante los mensajes de la televisión, pudiendo discriminar, seleccionar y evaluar los mensajes emitidos. En la segunda, es posible distinguir dos líneas de argumentación que se contraponen. Por una parte, está la visión negativa de la televisión según la cual, salvo algunos programas que caerían en la categoría de "culturales", no sería un aporte para los televidentes. Por otra, la televisión cumpliría diversas funciones entre las cuales destaca lo lúdico, asumido como válido y necesario.

Para los estudiantes que finalizan sus carreras en el área educación la diversificación temática y programática que ofrece la televisión es visualizada como recurso educativo. Ello, en tanto ofrece la posibilidad de construir canales de comunicación con los niños a partir de sus intereses y visionados, así como obtener ideas creativas y entretenidas para utilizarlas con fines académicos. Esta mirada se 
vincula con la etapa de formación en que se encuentran estos estudiantes, quienes al momento de realizar la investigación efectuaban sus prácticas profesionales en distintos establecimientos educacionales de la Región Metropolitana y, por lo tanto, se proyectan en su rol como docentes.

Los medios de comunicación, y en particular la televisión, son reconocidos y legitimados por los estudiantes como importantes fuentes de información, ocupando un lugar privilegiado en su vida cotidiana. Los jóvenes otorgan valor a la información obtenida a partir de la televisión, visión que sería compartida por sus docentes. Para unos y otros la información no solo proporciona una lectura actualizada de la realidad social, sino también, permitiría aplicar los conocimientos adquiridos en la universidad a nuevas situaciones y contextos, generando procesos reflexivos que resitúan dichos conocimientos. Por otra parte, el manejo de información permitiría a los jóvenes una mayor participación en el debate que se genera en el aula.

La necesidad de adquirir una formación para la recepción crítica de la prensa escrita aparece como fundamental en los jóvenes que están terminando carreras de educación. Desde esta mirada, el rol del docente debiera orientarse a que los estudiantes adquieran una comprensión acabada del perfil del periódico que se lee y desarrollen su capacidad de analizar e interpretar la información que este proporciona. El estudiante se concibe entonces como un receptor activo, con capacidad de interactuar con el medio y, por lo tanto, el proceso de recepción se torna central.

Pese a que el discurso de los jóvenes no revela el ejercicio de una práctica permanente y sistemática de lectura de la prensa escrita, esta es visualizada como una herramienta que ofrece amplias posibilidades educativas dentro y fuera del ámbito formal. Dicha valoración se traduce en lecturas intencionadas, de acuerdo a necesidades e intereses académicos específicos, así como a búsquedas de orden más general que responden a necesidades de otro tipo de aprendizajes considerados valiosos para la inserción social actual.

Los jóvenes reconocen en los docentes el ejercicio de una práctica orientada a vincular los contenidos programáticos de su cátedra con el acontecer nacional. De este modo, el uso de la prensa con fines educativos, pese a no ser generalizada, sería intencionada y valorada por los docentes como un instrumento útil que permite contex- 
tualizar las temáticas abordadas en la clase. Por otra parte, los estudiantes valoran la prensa en tanto les permite adquirir aprendizajes múltiples y responder a las expectativas del docente.

\section{Conclusiones}

Los resultados obtenidos a partir de esta investigación permiten concluir que los estudiantes poseerían valoraciones y significaciones del espacio educativo y los actores allí presentes, distintas a las que propone el sistema de educación superior; diferencias que no han sido incorporadas del todo por la institución universitaria. Los jóvenes desarrollan un proceso activo de interpretación a partir del cual perciben, se apropian, negocian y re-producen significados en torno a la experiencia educativa que los convoca. En dicha re-construcción, los significados hegemónicos propuestos por el sistema de educación superior son resignificados y adquieren nuevos y variados sentidos que solo pueden ser comprendidos en el contexto en el que tienen lugar.

En este marco, la institución de educación superior, el espacio educativo en el que cotidianamente se desarrolla la práctica pedagógica, la figura del docente, del grupo de pares y del estudiante, así como los distintos escenarios donde los jóvenes obtienen aprendizajes, admiten más de un significado. Se trata de un proceso complejo y transversal donde coexisten lecturas compartidas y asimetrías comunicativas respecto de las distintas dimensiones que conforman el proceso de enseñanza aprendizaje de los estudiantes.

La ruptura intergeneracional de la que nos habla Margaret Mead (2002) suscita un conflicto aún no develado en el sistema educativo, que tiene su origen en la concurrencia de docentes y estudiantes a un proceso de formación, desconociendo, unos y otros, el universo cultural desde el que se sitúa su contraparte. Dada la inexistencia de un reconocimiento mutuo que permita comprender la manera en que cada uno de estos actores se plantea el proceso de enseñanza - aprendizaje, persiste una definición del "sujeto de la educación" emanada, fundamentalmente, desde el sistema educativo y sobre la base de una perspectiva homogenizante, que ignora, tal como nos propone Reguillo (2000), el carácter cambiante y discontinuo que caracteriza a los jóvenes en su condición de sujetos sociales. Dicha perspectiva posterga la construcción personal que éstos realizan en 
forma permanente, recreando el sentido y significado del "ser estudiante universitario" y con ello, oculta las distintas formas en que éstos asumen su formación y se insertan en la universidad.

La universidad no constituye el único escenario al que los jóvenes atribuyen valor educativo. Tal como propone Pozo (1996), el contexto social en que hoy se sitúan provee de múltiples posibilidades para la adquisición de aprendizajes que éstos aprovechan y utilizan en su experiencia educativa, optimizando su proceso formativo.

Con relación a la institución universitaria, se advierte una diversidad de significaciones que, combinadas en distintas formas, transitan desde el compromiso con la sociedad y el bien común, hasta una mirada más instrumental centrada en el título profesional como fuente de inserción social y laboral. Los estudiantes se aproximan a la experiencia universitaria con toda la heterogeneidad y dinamismo que los caracteriza, adoptando una serie de prácticas comunicativas que tienen su base en los distintos significados que es posible advertir en el continuo entre los extremos descritos. Con todo, esta institución constituye una fuente de desarrollo personal que les proporciona herramientas que favorecen su relación con el medio social, con otros y consigo mismos.

Específicamente, en el caso de la Universidad Católica Silva Henríquez, la vocación de servicio que está a la base de su proyecto institucional y que dicha Casa de Estudios quiere proyectar hacia el conjunto de la comunidad universitaria, es recogida por los jóvenes, quiénes, con independencia de su postura religiosa, aprecian la orientación valórica que otorga "centralidad a la persona".

Este factor adquiere notoriedad en el discurso de los estudiantes y se expresa, entre otros aspectos, en el establecimiento de relaciones con sus docentes que legitiman la importancia del componente afectivo en la práctica pedagógica. Si bien esta mirada más integral del ser humano les permite sentirse acogidos en una institución que se aleja del imaginario frío e impersonal con que se asocia la universidad, tiende a evocarles un modelo escolarizante de educación.

Respecto de este punto, no es posible hablar de un modelo puro, se trata más bien de una situación híbrida que integra componentes de un modelo de comunicación y educación lineal y unidireccional, con una mirada que, siguiendo a Kaplún (1998a), ubica al estudiante 
como sujeto de su propio aprendizaje y a éste último como un proceso activo de construcción y recreación del conocimiento.

En este sentido, la experiencia adquirida por los estudiantes en el transcurso de los doce años que contempla su educación escolar formal se encuentra en permanente tensión con un sistema educativo que, por una parte, representa mayor complejidad y una ruptura con la cultura escolar y, por otra, refuerza las nociones adquiridas en su formación inicial.

Para los jóvenes la adquisición de aprendizajes se produce en la práctica, en escenarios diversos y con la participación de un conjunto de actores que la hacen posible. El aprendizaje deja de ser una actividad circunscrita a las paredes del aula ya que penetra todas las actividades sociales en que participan y, por lo tanto, todos los tiempos que configuran su experiencia cotidiana son tiempos de aprendizaje.

El rol del docente en el proceso de enseñanza aprendizaje es concebido en términos integrales. Ello implica que, en el ejercicio de la docencia, confluye una serie de factores de carácter ético, pedagógico y teórico que determinarán el grado de éxito o fracaso en el proceso de enseñanza aprendizaje que quiere intencionar.

De este modo, existe un desfase en la concepción que los jóvenes tienen sobre la figura del docente y aquella que propone el sistema de educación superior. La autoridad y respeto que hace algunas décadas eran conferidos al maestro por el sólo hecho de ocupar esta posición ya no es tal. Los destiempos de la educación a los que alude Martín Barbero (2002) se expresan, en este caso, en el cuestionamiento que la figura del docente tiene en el discurso de los jóvenes, en el sentido que ya no constituye un referente per se.

Los jóvenes asumen que en la formación universitaria debieran adoptar un rol más autónomo con respecto a su proceso de aprendizaje, en la práctica, esta representación adopta múltiples formas, íntimamente relacionadas con las circunstancias concretas que van marcando su paso por la universidad y, que en muchas oportunidades, determinan una orientación que se aproxima mucho más a un enfoque clásico de educación. Cuando las circunstancias a las que hacíamos alusión ponen en riesgo su éxito en el programa de formación del que participan, aspectos como la priorización de asig- 
naturas, la rentabilidad del tiempo en función de los resultados que se espera obtener, la concepción instrumental de la nota o el establecimiento de una relación pragmática con el docente se tornan prioritarios.

En este escenario, en su tránsito por la universidad, los estudiantes desarrollan una serie de prácticas comunicativas formales y no formales que, mediadas por la manera en que significan y dan sentido a su proceso de aprendizaje y su relación con la institución educativa, les permiten alcanzar metas de carácter académico y social.

Una de las disonancias comunicativas especialmente significativa en tanto representa mayor dificultad para los jóvenes en la asimilación de la cultura del sistema universitario, esta dada por la adquisición y dominio de un lenguaje que los valide en su desempeño académico. Retomando a Bernstein (2001) -si el código constituye el principio regulador, que selecciona e integra significados relevantes, formas de realización de los mismos y contextos evocadores y, el código académico o elaborado representa el tipo de lenguaje significado como propio de la universidad- entonces, éste último, constituiría la forma de comunicación legítima en este espacio. Así, al demarcarse los criterios que diferencian el código legitimo de aquel significado como irrelevante o inapropiado, el lenguaje cotidiano o código restringido al que se inclinan de manera natural los estudiantes es desestimado, generándoles dificultades en su proceso de enseñanza - aprendizaje.

Con relación a las prácticas comunicativas formales y no formales que tienen valor de aprendizaje para los estudiantes, se puede afirmar que los jóvenes desarrollan una serie de prácticas comunicativas y habitan diversos espacios donde obtienen aprendizajes múltiples que complementan la formación que reciben en la universidad. De esta forma, la noción de currículum transversal a la que alude Hopenhayn (2003) se cristaliza en una experiencia que concibe el aprendizaje como un continuo que atraviesa los distintos escenarios que habitan y donde lo académico y lo lúdico forman parte de un todo que hace posible la síntesis de lo aprendido.

Finalmente, respecto de la inserción de los jóvenes en los medios de comunicación masiva y las nuevas tecnologías de la comunicación y la información, es posible subrayar el valor educativo que éstos le atribuyen. No obstante lo anterior, Internet es la tecnología que 
representa el mayor aporte educativo para los jóvenes, quienes participan de la "cultura virtual", incorporando una serie de prácticas comunicativas.

Por otra parte, el discurso de los jóvenes deja en evidencia la existencia de la brecha generacional que el uso de Internet produce entre estudiantes y docentes. Estos últimos dan a Internet un uso que refleja una inserción tardía y parcial que no lo intenciona necesariamente como recurso educativo.

Respecto del impacto de internet en la educación es importante señalar que los jóvenes lo consideran un complemento a las actividades educativas, que en ningún caso sustituiría el aula como unidad de acción espacio - temporal, privilegiada para el diálogo y la figura del docente como mediador del conocimiento.

Estos resultados confirman que los desafíos que la emergencia del siglo XXI plantea a las instituciones de educación superior, no solo le exigen revisar el tipo de relaciones que establece con el gobierno, la sociedad civil y en general con el contexto internacional, sino también y, muy especialmente, con uno de los actores que dan sentido a su existencia, a saber, los estudiantes universitarios.

La noción de educación que queremos intencionar deja atrás los significados de transmisión y acumulación de información y/o conocimientos presentes hasta nuestros días, orientándose a favorecer en los estudiantes la capacidad de gestionarlos y aprovecharlos para toda la vida, esto es, en virtud de su adaptación al contexto actual, personal y social que les toca vivir.

Ello implica un desafío para quienes imparten docencia en términos de resignificar sus estrategias didácticas, así como los sistemas de comunicación y distribución de los materiales de aprendizaje que es necesario intencionar.

\section{Bibliografía}

Bernstein, Basil (2001). "Educación, Mercado e Identidades Pedagógicas Emergentes". En: Persona y sociedad, Volumen XII No 2-3, Universidad Jesuita Alberto Hurtado, Instituto Latinoamericano de Doctrina y Estudios Sociales ILADES. 
Brunner, José Joaquín y Tedesco, Juan Carlos (editores) (2003).

Las Nuevas Tecnologías y el Futuro de la Educación. Instituto Internacional de Planeamiento de la Educación (IIPE) - UNESCO y Septiembre Grupo Editor, Buenos Aires Argentina.

Brunner, José Joaquín (2000).

Educación: Escenarios de Futuro: Nuevas Tecnologías y Sociedad de la Información. Programa de Promoción de la Reforma Educativa en América Latina y el Caribe.

Delors, Jacques (1996). La Educación Encierra un Tesoro, Informe a la UNES$\mathrm{CO}$ de la Comisión Internacional sobre la Educación para el siglo XXI, Santillana Ediciones UNESCO.

Fontcuberta, Mar (2003). “Medios de Comunicación y Gestión del Conocimiento". Revista Iberoamericana de Educación $\mathrm{N}^{\circ} 32$.

Hopenhayn, Martín (2003).

Educación, Comunicación y Cultura en la Sociedad de la Información: Una perspectiva Latinoamericana. Serie Informes y Estudios Especiales $N^{\circ} 12$, CEPAL, Santiago de Chile.

Huergo, Jorge (2001). C Comunicación/Educación, Ámbitos, Prácticas y Perspectivas. Ediciones de Periodismo y comunicación, Universidad Nacional de la Plata, Buenos Aires Argentina.

Instituto Nacional de la Juventud (1999).

Tercera Encuesta Nacional de Juventud, Situación y Condición de los Jóvenes Chilenos a Comienzos de Nuevo Siglo. Gobierno de Chile.

Kaplún, Mario (1998a). Una Pedagogía de la comunicación. “Énfasis en el Proceso". Ediciones de la Torre, Madrid.

Kaplún Mario (1998b). "Procesos Educativos y canales de Comunicación", Chasqui Nº 64.

Martín-Barbero, Jesús (2002).

Oficio de Cartógrafo Travesías Latinoamericanas de la Comunicación en la Cultura. Fondo de Cultura Económica. México. 
Martín-Barbero, Jesús (2003).

"Saberes Hoy: Diseminaciones, Competencias y Transversalidades", Revista Iberoamericana de Educación $\mathrm{N}^{\circ} 32$.

Mead Margaret, en Martín-Barbero, Jesús (2003).

"Oficio de Cartógrafo Travesías Latinoamericanas de la Comunicación en la Cultura". Fondo de Cultura Económica. México.

Monereo Carles y Pozo Ignacio (2003).

La Universidad Ante la Nueva Cultura Educativa: Enseñar y Aprender para la Autonomía. Síntesis. Madrid.

Pozo, Ignacio (1996). Aprendices y Maestros: La Nueva Cultura del Aprendizaje, Psicología y educación. Alianza. Madrid.

Quiroz, María Teresa (2003).

"Aprendizaje y Comunicación en el siglo XXI". Enciclopedia Latinoamericana de Sociocultura y Comunicación, Norma, Buenos Aires.

Reguillo Rossana (2000). Emergencia de Culturas Juveniles: Estrategias del Desencanto, Norma, Buenos Aires. 